\title{
ADAPTIVE TECHNOLOGY APPLICATION FOR VIBRATION-BASED DIAGNOSTICS OF ROLLER BEARINGS ON INDUSTRIAL PLANTS
}

\author{
Aleksey Mironov ${ }^{1}$, Pavel Doronkin $^{1}$, Alexander Priklonskiy ${ }^{1}$, Sergey Yunusov $^{2}$ \\ ${ }^{I} D$ un $D$ centrs Ltd. \\ Margrietas Str. 7, Riga, LV-1046, Latvia \\ Phone: +37167468850. Fax +37167468856 \\ E-mails:aleksei@ddcentrs.lv,pavel@ddcentrs.lv,pai_ev@inbox.lv \\ ${ }^{2}$ Transport and Telecommunication Institute \\ Lomonosov Str. 1, Riga, LV-1019, Latvia \\ Phone:+37126435655.E-mail:yunusov@inbox.lv
}

\begin{abstract}
Roller bearings are widely used in equipment of different applications; therefore, the issues related to the assessment of bearing technical state and localization of bearing faults are quite important and relevant. The reason is that technical state of a bearing is a critical component, which determines efficiency of a mechanism or equipment. For bearings inspection and diagnostics, various methods of vibration-based diagnostics are used. The adaptive technology for vibration-based diagnostics developed in ,D un D centrs” is an effective tool for evaluation of technical state of bearings in operation compared to the existing SKF method.
\end{abstract}

Keywords: roller bearing, vibration-based diagnostics, SKF method, deterioration, adaptive technology

\section{Introduction}

Many industrial plants comprise rotor-type mechanisms such as electric motors, pumps, fans, turbines, generators etc. operating at high rotation frequencies and, therefore, using roller bearings in their design accordingly. Being high-precision mechanisms, they can operate for many years under favourable operating conditions. However, roller bearings cannot use their potential resource in full for various reasons like severe operating conditions and the established maintenance strategies. On the one hand, any failure of a bearing may lead to a long-lasting downtime of equipment and economic losses. On the other hand, bearings are often replaced in order "to be on the safe side", leaving a great portion of the resource unrealized.

The problem can be solved by transition to condition-based maintenance, which requires improvement of instrumentation and diagnostic methods development. To this end, methods of vibrationbased diagnostics are used as the most efficient ones for evaluation and prediction of technical state. The advantage of vibration-based diagnostics is that it allows arranging the operating equipment technical state monitoring without affecting production cycle.

Currently, there is a consistent trend of transition to condition-based maintenance of equipment, aimed at operating costs cutting down and improving the equipment efficiency without any loss in its reliability, ensuring the required safety level at the same time. A necessary pre-requisite for the implementation of condition-based maintenance is the availability of monitoring and diagnostics means which would allow timely and precisely determine the technical state of the object, therefore, precluding its failure.

\section{Vibration-Based Diagnostics of Roller Bearings Traditional Methods Efficiency}

Roller bearings having faults on bearing tracks, balls, or bearing cage generate some additional dynamic forces which are transferred onto the outside bearing housing assembly and the surrounding structure. These forces can be periodic or stochastic by nature and appear mainly within high-frequency vibration range. However, it is quite difficult to identify any fault symptoms of a bearing inside of various mechanisms and machines using vibration characteristics due to the fact that, apart from the bearings, 
other machine assemblies act as vibration sources, too. Nonlinear interaction of a set of vibration sources produces a complicated picture of fluctuations at the measurement point on bearing housing assembly.

The main approach used in traditional, vibration-based methods for bearing diagnostics is based on the relationship between the frequencies of typical components of vibration spectrum and the commontype defects of a roller bearing. The presence and the intensity of defects in a bearing are estimated by composition and ratio of spectral components of the measured directly or post-processed vibration signal.

There exist various methods for analyzing vibration of roller bearings, aimed at the solution of the two main problems: 1) extracting the bearing-generated signal, thereby separating it from other aggregates, and minimizing "noise", that may conceal the bearing-generated signal - especially at early stages of the fault propagation, and 2) identifying the technical state of the bearing - i.e., distinguish between generally normal bearings and defective ones, defining faulty components of the latter. Frequency domain analysis is the most widely-used method of localization of bearings' faults. Most of the methods of this category analyze energy spectrum of vibration signal using Fast Fourier Transform which has some specific shortcomings (Barkova, 2000).

The non-uniformity of spectral behaviour when approaching a state of emergency (Barkov et al.) requires some more efficient diagnosing methods - in particular, those based on demodulation of highfrequency random vibration waveform envelope. The vibration envelope method analyses only the vibration power of components of the same nature which are preliminary extracted from vibration signal. If the extracted part of vibration has many different components with an impact-type dominating component available - the power of the extracted vibration components is changed by periodical bursts. Then, a large number of multiple harmonics missing in the spectrum of a high-frequency vibration signal occurs in the spectrum of power fluctuations of high frequency vibration. The analysis of power fluctuations of preliminary extracted vibration components of similar nature is exactly the vibration envelope spectral analysis. However, the spectral analysis of vibration envelope is sometimes construed not as power fluctuations analysis but rather as the analysis of root-mean-square value of high-frequency vibration, which in principle doesn't change the physical basis of obtained results in any way.

Usage of vibration enveloping method can effectively solve the problem of diagnosis of roller bearings and other components of rotary machines (Barkov, 2000) operating mainly at low rotation speeds. At the same time, this method has some bottlenecks - like for instance, different flaw sensitivity of bearing elements, which determines different hazard thresholds of defect populations; the existence of a few progressing defects desensitizes the method etc.

Bearing monitoring and diagnostics tools are developed by many companies and organizations, and one of the leaders in this field is the Swedish $S K F$ - a leading global supplier of products and services associated with roller bearings. Within the framework of the study carried out on industrial plants to assess the technical state of their roller bearings, an SKF-manufactured device CMAS 100-5L was used (Fig. 1).

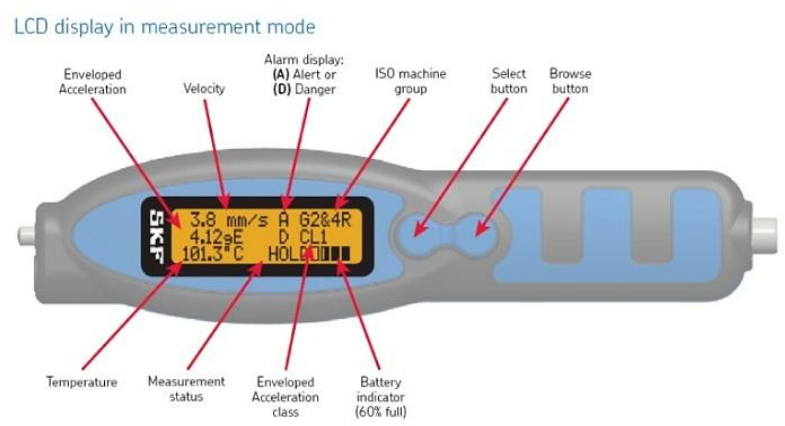

Figure 1. SKF Machine Condition Advisor CMAS-100

In this device, the measurement of a couple of testing parameters has been implemented: the absolute level of machine vibration in the low-frequency range and the vibration envelope acceleration value.

In the course of the study, the technical state of 12 bearings from various industrial plants, including those of RigasUdens - the main water supplier of the city of Riga - was analyzed.

The study of the bearings of each plant was carried out in several stages. Initially, the instrumental monitoring of plant vibration on the bearing housing assembly was carried out using CMAS 100-5L 
device. Then, the bearings were dismantled for the subsequent fault localization. On completion of maintenance of the plant, a new bearing of the same type as the dismantled one was installed therein. After the plant maintenance and putting it into operating mode, the vibration was measured again by CMAS 100-5L on the bearing housing assembly (at the same point where the vibration of the replaced bearing was measured).

According to the fault detection results, all the bearings were divided into two groups. The first group included the bearings with minor wear in the form of visible traces of bearing tracks of uniform character. The second group included bearings with substantial damage in the form of spots of varying size and depth over $0.05 \mathrm{~mm}$. To assess the extent of damage of the bearing tracks, the bearing track damage ratio was introduced, which characterizes the relative length of the damage:

$k_{d}=l / \pi d$,

where $l$ is the cumulative length of damaged sections of bearing track;

$d$ - diameter of bearing track.

The damage ratios, calculated for bearing elements, have shown that the damage extent of the elements may differ substantially. For example, with respect to one bearing, $k_{d}$ for the bearing inner race is $60 \%$, while $k_{d}$ for the bearing outer race is $5 \%$ and for roller elements (rollers) - $0 \%$ (Fig. 2).

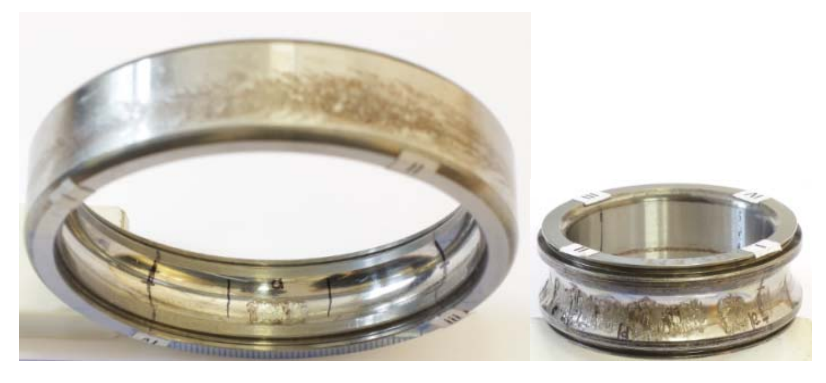

Figure 2. Faults of the inner and the outer race of bearing

To form bearing groups with substantial and insubstantial faults, a complex damage ratio was introduced, which takes into account intensity (relative depth) $i=d e p t h / 50$ um of the damaged section of bearing track (in um ):

$k_{d}^{i}=20 \lg \left(i^{2} d\right)$

The damage ratio diagrams describing two groups of bearings - those with substantial and insubstantial wear - are presented on Figure 3.

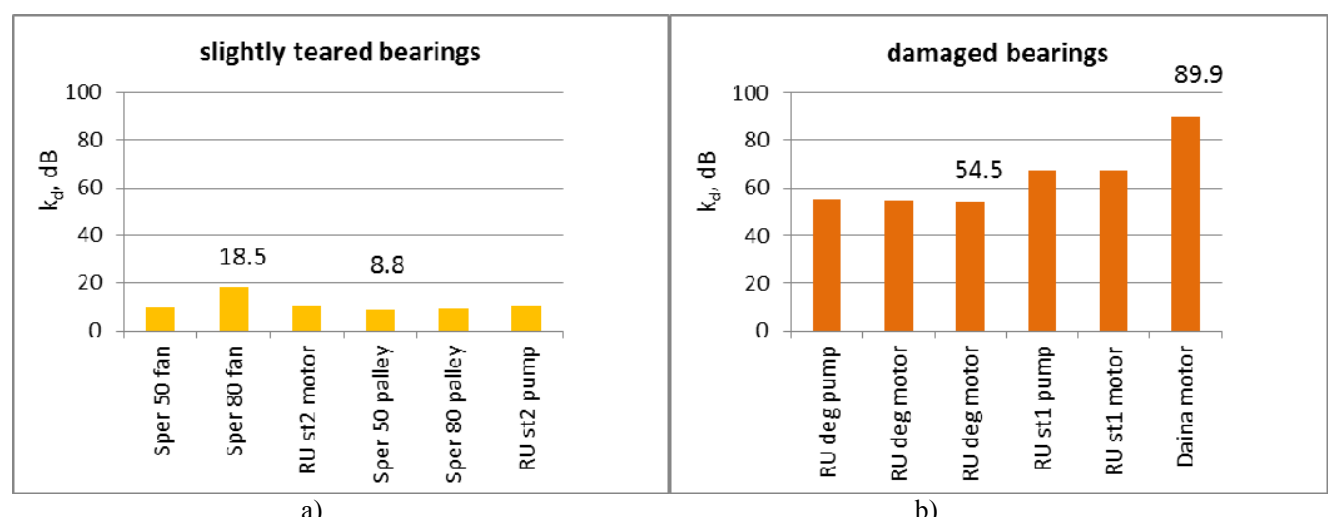

Figure 3. Bearings split-up by groups: a) those with insubstantial wear; b) those with substantial wear 
As it can be seen from the diagrams, the bearings with substantial wear are characterized by the value $k_{d}^{i}$ within the range $54 \ldots 90 \mathrm{~dB}$; with respect to the bearings with insubstantial wear $k_{d}^{i}$ lies within the range $8 \ldots 20 \mathrm{~dB}$.

The efficiency of the parameters measured by SKF device was assessed by analysing diagnostic messages from the device and numerical values of the parameters measured by it, with the subsequent comparison with the calculated assessments of actual faults stated above. The device produced diagnostic messages of two kinds: "Alert" - recommendation to halt the aggregate and investigate it, and "Danger" the message signalizing about a fault of the bearing.

The results of measurement of the two SKF parameters at all the investigated objects are shown on Figure 4 and 5.

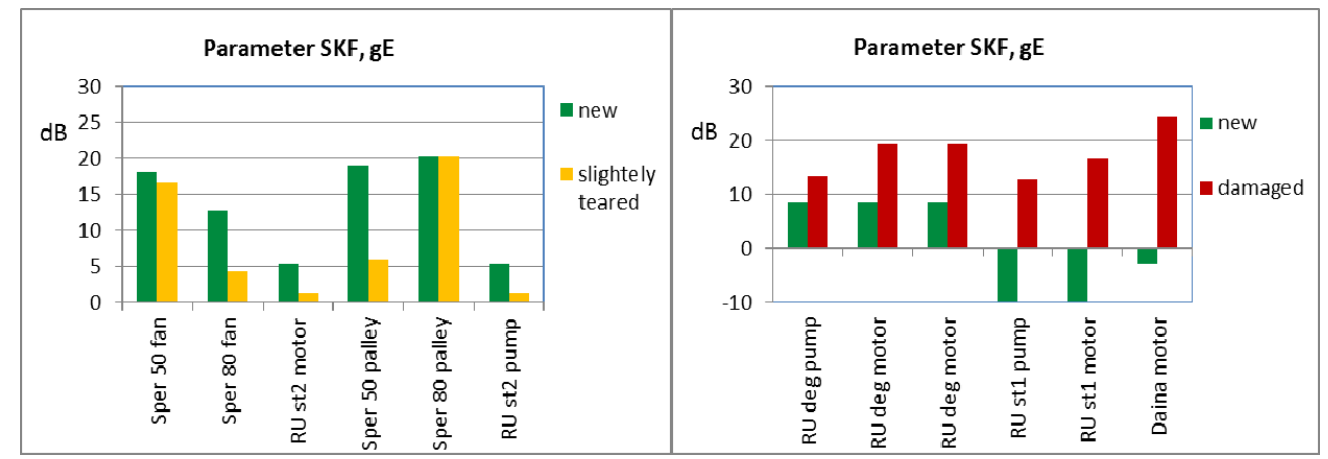

a)

b)

Figure 4. The results of SKF-performed measurement of $\mathbf{g E}$ parameter of the bearings split up by groups: a) with insubstantial wear; b) with substantial wear

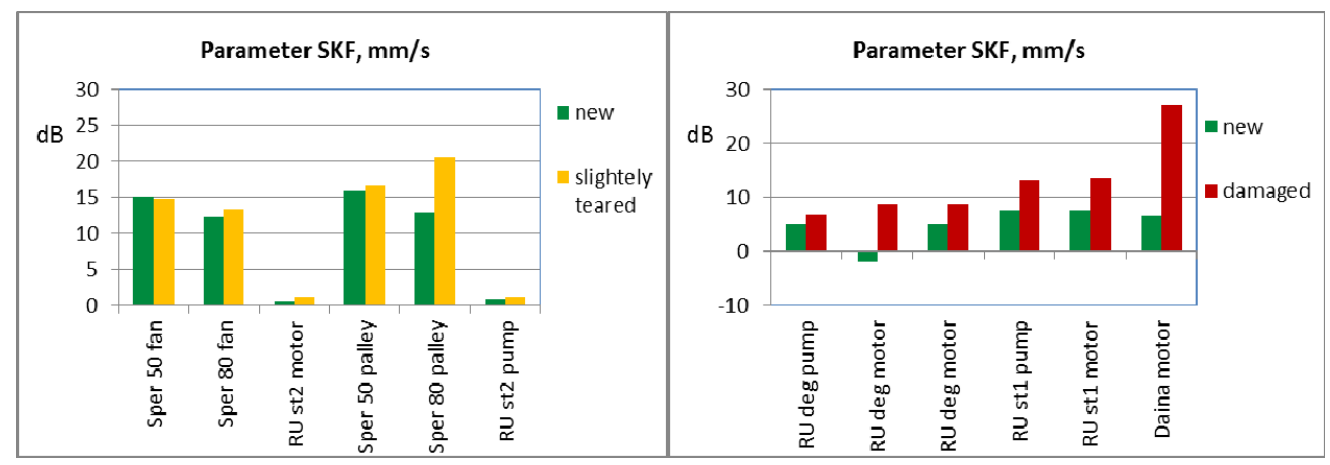

a)

b)

Figure 5. The results of SKF-performed measurement of $\mathbf{m m} / \mathbf{s}$ parameter of the bearings split up by groups: a) with insubstantial wear; b) with substantial wear

To facilitate the comparison with fault detection results, the SKF-performed measurement data are presented in decibel scale.

Comparing the bearing fault detection results and the measurement data obtained by SKF device, one may come to the conclusion that the device has made errors of first kind (false alarm) and second kind (non-detection).

For example, in 12 tests where technically sound bearings were investigated, SKF device had identified presence of faults or their probability in brand-new bearings, in particular: the device had reported the occurrence of a fault three times (Danger) and once - by issuing breakdown prevention signal (Alert). When the group of 6 used bearings was investigated where no visible defects were found after the dismantling (i.e. the bearings were sound), the device issued Danger twice and Alert - once. Therefore, the device incorrectly identified technical state of bearings in 7 cases out of 18, i.e., the false response percentage of the device made up about 39\% accordingly.

The device has shown the following results of operation with the group of bearings having substantial faults. In process of investigation of 6 bearings with substantial faults, the device has made 
the correct diagnosis thrice: it suggested preventive maintenance (Alert) twice - with respect to bearings which have shown traces of corrosion indeed in process of their dismantling; once, the device has highlighted a defect (Danger) which was confirmed when the bearing fault detection took place. As regards the remaining three bearings with substantial defects, the device has even failed to issue the signal Alert, which may be interpreted as defect skipping. Therefore, with respect to that group of bearings, the probability of correct identification of their technical state by SKF device constituted $50 \%$ only.

The total number of test runs performed by SKF device was 24 (12 of them were performed for testing used bearings, and 12 - for testing new ones). Based on the yielded results, 10 errors were recorded, 7 of which were false response with technically sound bearings (error of the $1^{\text {st }}$ kind) and $3-$ "non-detection of target" (error of the $2^{\text {nd }}$ kind) with the bearings having substantial defects which may lead to the bearing's failure in future. In this case, the error of the $2^{\text {nd }}$ kind constituted $40 \%$ which is generally intolerable to ensure reliability of the plant.

These results suggest that the vibration analysis methods used in SKF device cannot be considered acceptable, since a high percentage of errors of the first and the second kind leads to unsubstantiated prevention maintenance jobs and uncontrolled failures, which ultimately increases the operating costs for the plant maintenance.

One reason of the low efficiency of roller bearings' technical state identification by SKF device is dependence of measured parameters $(\mathrm{gE}$ and $\mathrm{mm} / \mathrm{s})$ from the design features of the plant. The diagrams (Figs. 4a and 5a) clearly show how far the measured parameter levels differ from each other for new bearings (or those with minor damage) in different industrial plants. It can be seen from Fig. 4a that $g E$ parameter obtained through the envelope monitoring method was often smaller for bearings having a minor wear than for technically sound bearings. We should note at this point that the $\mathrm{mm} / \mathrm{s}$ parameter compatibility between new bearings and those with minor wear (Fig. 5a) was sensibly better. The values shown by the bearings with a minor wear were a little higher than those shown by the new bearings. However, this parameter which is the generalized vibration parameter related to the plant as a whole does not highlight vibration of bearing and, therefore, it become non-diagnostic if other vibration sources present.

As regards the bearings with substantial defects, SKF parameters of defective bearings were higher than those of the new ones (Figs. $4 b$ and $5 b$ ) in terms of the two parameters. It should be noted at the same time that all the defective bearings made such a loud noise that it could be heard even without the device.

The method applied in SKF devices is quite fit for the bearings technical state monitoring, provided, however, that any change in the state of a bearing is controlled by comparing of the current vibration parameters with the values recorded at the beginning of their operation. However, initial values of vibration parameters corresponding to the beginning of operation are missing as a rule and it may impose constraints on the method usage with respect to millions of bearings already in operation. Thus, bearing diagnosis should be based on one-time measurements of the parameters providing reliability of making decision on defect appearance. Therefore, in order to unify the application of SKF method to all bearings without taking their prehistory into account, it is necessary to consider the SKF parameter spread, which happens due to the design features of different units.

The diagrams (Fig. 6) show the correlation between used bearings parameter values (in red) and the calculated value (in grey), which takes into account parameter spread with respect to values characterizing new bearings in different units. In cases where the red values are substantially higher than the grey ones, defect identification is ensured; otherwise, an error is possible.

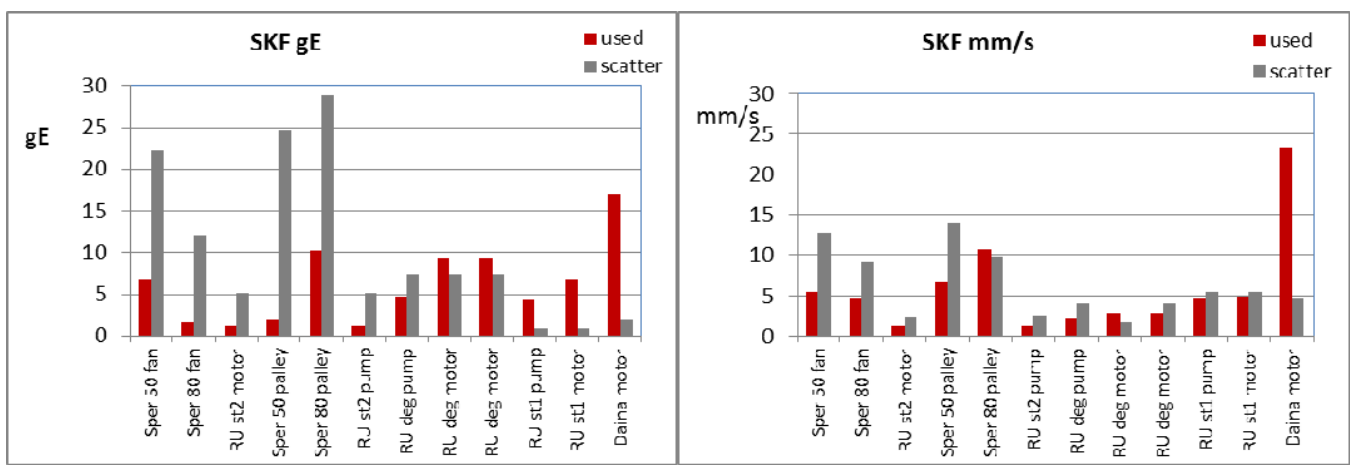

a)

b)

Figure 6. Measured values of parameter $\mathbf{g E}$ (a) and $\mathbf{m m} / \mathbf{s}$ (b) of waste bearings and the parameters spread with respect to new bearings 
As can be seen from the diagrams, the boldface identification based on $\mathrm{mm} / \mathrm{s}$ parameter was possible only with regard to the bearing having the largest faults. The $g E$ parameter ensured a reliable identification with respect to three bearings having the largest defects. However, in all other cases, both parameters were not able to identify the defect with confidence. The obtained results of the bearings technical state diagnosing using SKF device attest to the fact that the method used is not efficient enough; therefore, new methods aimed at enhancing the certainty of bearings diagnosis should be developed, or the existing methods of the type should be improved.

As an alternative to the existing methods, an adaptive technology for vibration-based diagnostics of roller bearings, developed in ,D un D centrs" was evaluated. The description of the technology and the obtained findings are stated in the next section.

\section{Adaptive Technology for Roller Bearings Technical State Monitoring}

\subsection{The concept of adaptive technology for bearing monitoring}

The adaptive technology for bearing monitoring bases on a joint processing of vibration signals synchronized with rotation speed, where kinematic parameters of a bearing are taken into account. The latter, in turn, are determined by the number and diameter of balls (rolling elements) and by the diameters of bearing tracks of the inner and the outer race. Figure 7 presents the example of measurement set up applied in the adaptive technology.

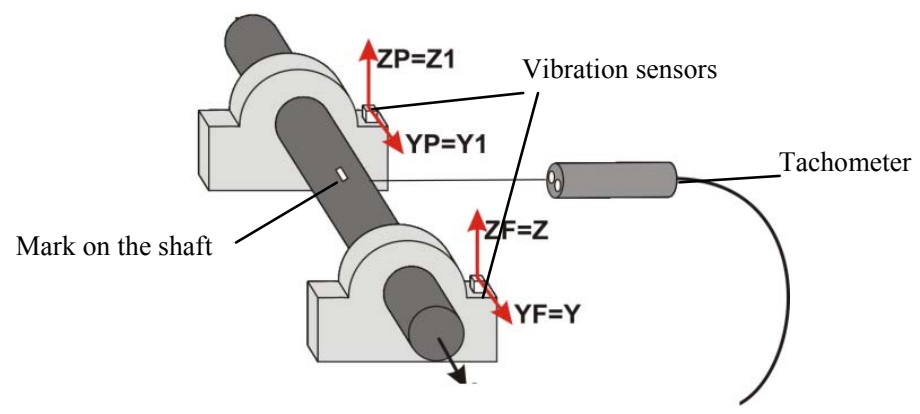

Figure 7. The adaptive technology parameters measurement diagram

Recorded vibration signals and the rotation speed data is processed according to the methods developed in ,D un D centrs".

The main feature of this method is that synchronous enhancing and averaging of signals is carried out in the time domain. Structural normalization of vibration signal is another feature of the method. The adaptive technology for vibration-based diagnostics of a bearing separately estimates states of inner and outer races as well as the balls (rolling elements). At the same time, due to normalization, the obtained estimates become indifferent towards the design features of the unit. The technique uses variational approach towards data processing, which allows one to make the technology insensitive to the bearing operation conditions the effect of which has a major impact on the vibration characteristics of any unit. Variational approach is used to improve the resolution of the method with respect to each component by calculating two or more options to assess the testing parameter, each of which is based on different assumptions about the scale of the alleged defect. Since the scale of a possible defect is not known a priori, the defect identification probability will be higher if the calculation is based on the assumption of two or more expected scales of the alleged defect. For example, in the study, sizes (scales) of hypothetical defects equal to $0.5 \mathrm{~mm}$ and $5 \mathrm{~mm}$ were selected respectively, serving subsequently as a basis for calculation of the testing parameter values for each component of the new and the waste bearings of the unit.

The graphs below (Figs. 8 and 9) show estimated state parameters (in decibel scale) calculated by adaptive method for plants, one of which comprised a bearing with minor wear, while the other a bearing with significant wear. The numerical estimate of the inner race state is marked with blue on the diagram, while that of the outer race state is marked with maroon and that of the balls is marked with green. 

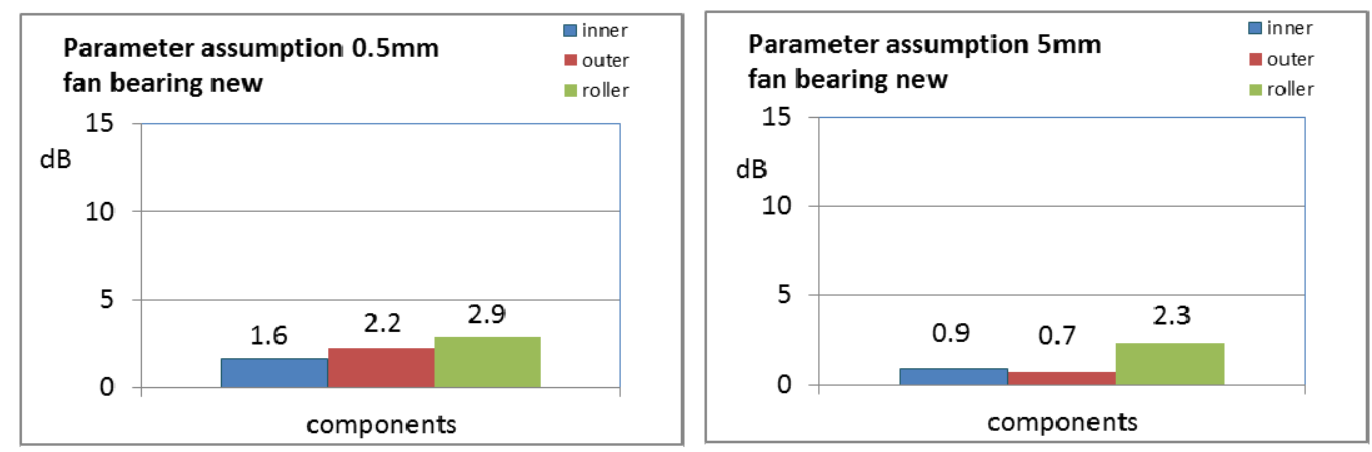

a)
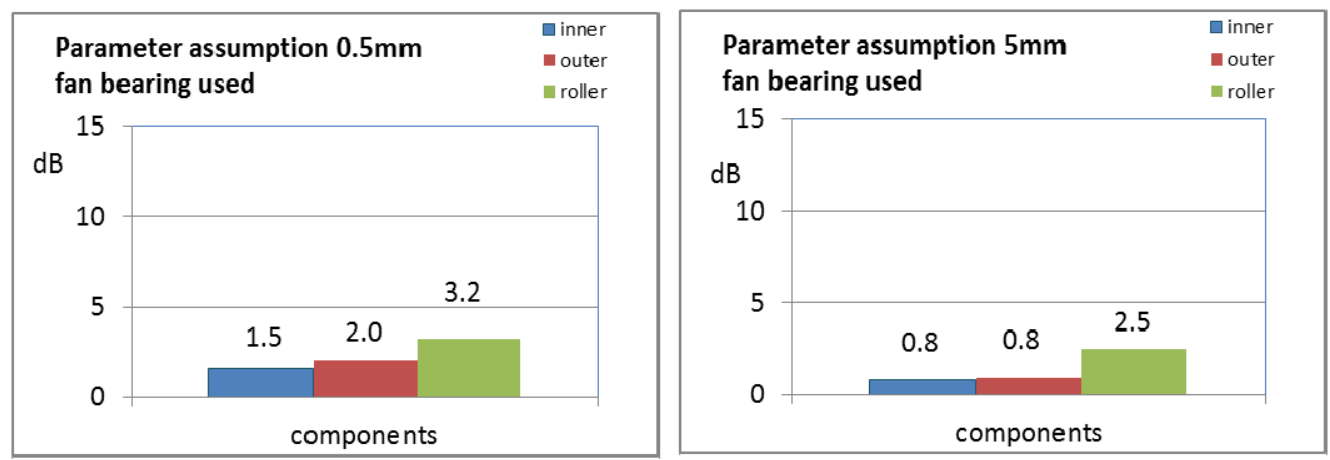

b)

Figure 8. Results of calculation of testing parameter with respect to the plant with minor wear of bearing: a) - new; b) - used

The calculation results presented on Figure 8 show small differences of parameter values $(0.2-0.3 \mathrm{~dB})$ between the components of the new and the used bearing, which actually corresponds to their initial technical condition.

The diagrams of Figure 9 show that parameter values of the inner race of the faulty bearing are larger than those of the new bearing installed into the same unit. In terms of small-scale defects $(0.5 \mathrm{~mm})$ the difference in values constituted $8.4 \mathrm{~dB}$, while in terms of the scale $5 \mathrm{~mm}$, it constituted $10.8 \mathrm{~dB}$.
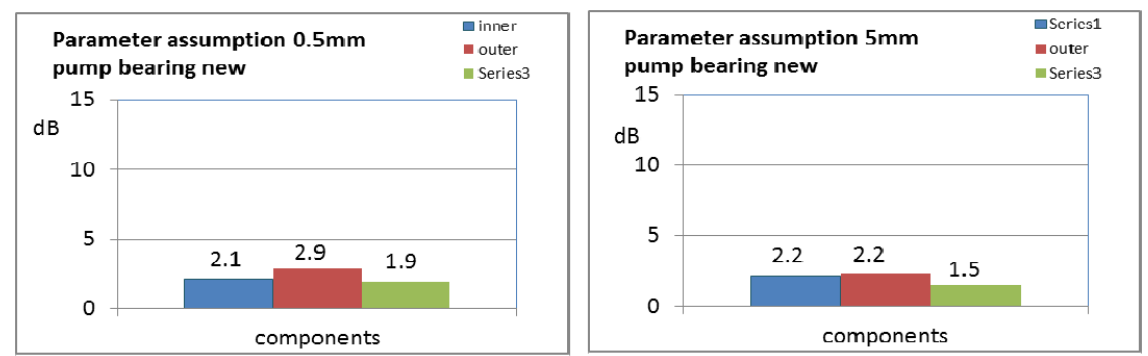

a)
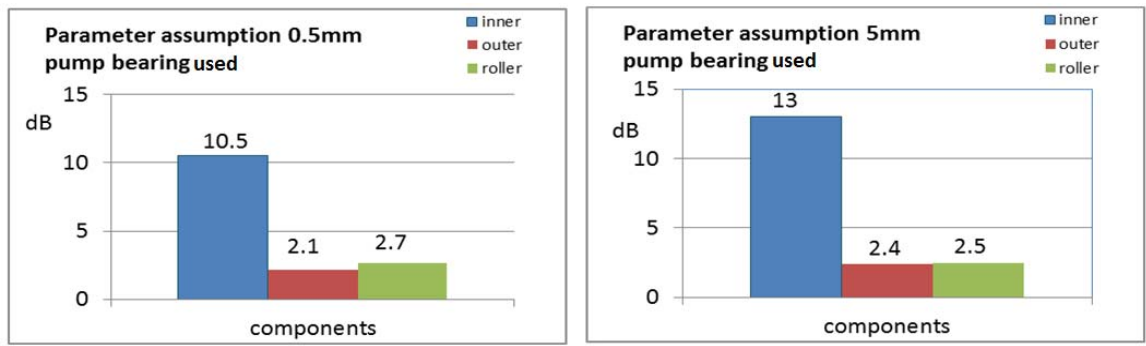

b)

Figure 9. Testing parameter calculation results for the unit with a substantial wear of the bearing: a) - new; b) - used 
The obtained estimates correspond to small changes of balls (rolling elements) and the outer race state, and to substantial changes in the condition of the inner race. The inner race parameter changes, nearly similar in terms of scale, attest to intermediate (between 0.5 and $5 \mathrm{~mm}$ ) scale of defects. Fault detection results (Fig. 2) had confirmed calculated estimates.

Therefore, state parameters of bearing components obtained by the adaptive technology enable one not only to identify bearing's faults at early stages of their propagation, but also to estimate their scale and identify the bearing part being most susceptible to deterioration.

\subsection{Evaluating efficiency of the method of adaptive technology for bearings monitoring}

To compare the efficiency of the adaptive technology and SKF method for monitoring there are state parameters obtained by both methods are collected in table 1. Samples collection is 12 technically sound bearing in start of its operation.

Table 1. Testing parameter values describing new bearings of units under study

\begin{tabular}{|c|c|c|c|}
\hline Parameter & Comb adaptive & \multicolumn{2}{|c|}{ SKF } \\
\hline Object & $\mathrm{dB}$ & " & "gE", dB \\
\hline Sper 50 fan & 7,6 & 15,1 & 18,1 \\
\hline Sper 80 fan & 9,3 & 12,3 & 12,8 \\
\hline RU st 2 motor & 7,0 & 0,6 & 5,4 \\
\hline Sper 50 palley & 7,4 & 16,0 & 19,0 \\
\hline Sper 80 palley & 10,6 & 12,9 & 20,3 \\
\hline RU st2 pump & 6,8 & 0,8 & 5,4 \\
\hline RU deg pump & 7,3 & 5,1 & 8,5 \\
\hline RU deg motor & 7,5 & $-1,9$ & 8,5 \\
\hline RU deg motor & 7,5 & 5,1 & 8,5 \\
\hline RU st1 pump & 10,9 & 7,7 & $-9,9$ \\
\hline RU st1 motor & 9,4 & 7,7 & $-9,9$ \\
\hline Daina motor & 5,3 & 6,7 & $-2,7$ \\
\hline Average & 8,0 & 7,3 & 7,0 \\
\hline \multicolumn{4}{|c|}{ Criteria } \\
\hline Variation, $\mathrm{dB}$ & 5,6 & 17,9 & 30,2 \\
\hline Max/Average, dB & 2,8 & 8,6 & 13,3 \\
\hline
\end{tabular}
calculated.

To assess the state parameters spread in $\mathrm{dB}$, two criteria - Variation and Max/Average - are

The parameter spread served as the criterion of information content: the larger the spread, the less informative is the parameter. Comparative assessment of efficiency is made for bearings that are in the same condition, technically sound.

In SKF method, two state parameters $-g E$ and $\mathrm{mm} / \mathrm{s}$ - are used, while the adaptive technology method uses the parameter comb adaptive for integrated assessment of a bearing as a whole. This parameter has the dimensions in $\mathrm{dB}$ and is the sum of parameter values of the three components of the bearing (inner and outer races and rolling elements):

$A_{\text {comb }}=A_{\text {ir }}+A_{\text {or }}+A_{b}$,

where $A_{\text {comb }} A_{\text {comb }}$ - integral testing parameter of adaptive technology;

$A_{i r}$ - testing parameter of inner race;

$A_{o r}$ - testing parameter of outer race;

$A_{b}$ - testing parameters of rolling elements.

Table 1 shows the comparison of SKF state parameter values (converted into $\mathrm{dB}$ ) and those of the adaptive technology for the units on which the study was conducted. Under the baseline measurements and calculations, their calculated average value is presented.

The last two rows in the table are the spread assessment criteria - Variation and Max/Average, which can evaluate the efficiency of SKF method and that of the adaptive technology method. 
The smaller the value of these criteria, the more efficient is the method and the more informative is the testing parameter.

The table shows that the SKF parameter spread greatly exceeds the integral testing parameter spread. Thus, the spread of parameter " $\mathrm{mm} / \mathrm{s}$ " exceeds the parameter comb adaptive spread by more than 4 times $(12.3 \mathrm{~dB})$, while the spread of " $\mathrm{gE}$ " parameter exceeds it by more than 17 times $(24.6 \mathrm{~dB})$.

For a more detailed comparison between the resolutions of the two methods, parameter Max / Average was used, which shows how far the scatter of each of the parameters exceeds its average value. Such a ratio provides a rough idea of parameter sensitivity of the bearing condition monitoring, namely, how far the value of the parameter corresponding to the defect should exceed the average value of the serviceable condition to ensure that the probability of a correct diagnosis would be higher than the error probability. As can be seen from the Table, it would be sufficient for comb adaptive parameter to provide the $40 \%(2.8 \mathrm{~dB})$ parameter increase in relation to the new bearing parameter value to identify a defect with confidence.

At the same time, with regard to SKF parameter, the " $\mathrm{mm} / \mathrm{s}$ " parameter should increase by 2.4 times and SKF " $\mathrm{gE}$ " - by 2.8 times.

To compare the methods more in detail, we will illustrate the difference in their resolution with the aid of diagrams showing the relation of values corresponding to new and used bearings of the same units (Fig. 10).

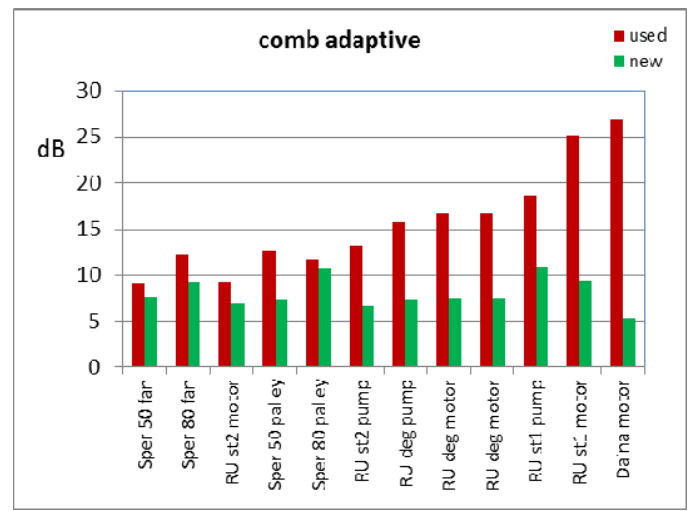

Figure 10. Relation of comb adaptive status parameter values for the used and the new bearings

Unlike SKF parameters (see Figure 6), the comb adaptive parameter values corresponding to all of the observed units with used bearings were higher than those corresponding to the same units with new bearings. Such a relation definitely ensures monitoring of the units' bearings, i.e., makes it possible to perform in-service inspection of their state. Importantly, the parameter value propagation areas for the new bearings (marked by green) $-5 \ldots .11 \mathrm{~dB}$ - and those with the used bearings (maroon) $-9 \ldots 27 \mathrm{~dB}$ almost do not overlap with respect to bearings of different types. The "overlapping" is observed only with three used bearings which had no defects whatever.

Now, let us analyse the relation of comb adaptive parameter values for used bearings and the calculated parameter spread values for new bearings (Fig. 11). Obviously, all bearings with major defects (six objects on the right) have the parameter values are at least by $6 \mathrm{~dB}$ (twice) higher than those of the new bearings.

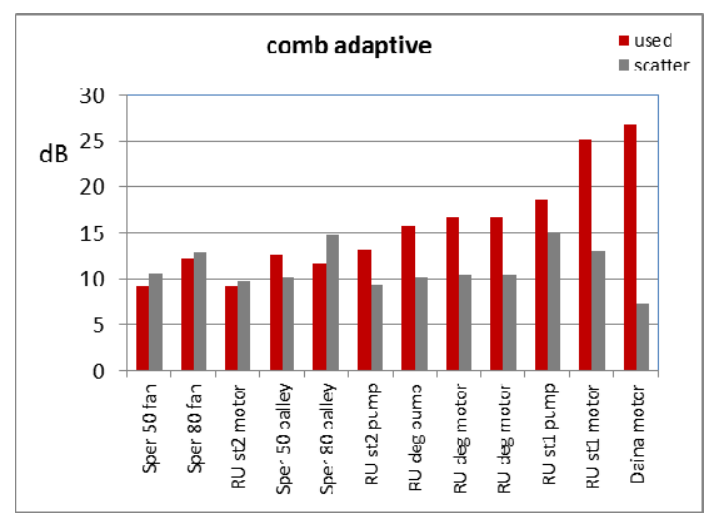

Figure 11. Relation between comb adaptive parameter values corresponding to units for used bearings and the calculated values of parameter spread for new bearings 
Thus, the difference between the parameter values of the adaptive technology related to healthy and defective bearings allows one not only to monitor individual objects reliably but to identify bearing defects in any unit with a sufficient degree of confidence regardless of the unit design and operating conditions.

\section{Conclusions}

The adaptive technology for monitoring roller bearings developed by „D un $D$ centrs” is an effective tool for assessing the technical condition of bearings in operation. This technology allows transition to condition-based maintenance of industrial installations, thereby ensuring reduction of unreasonable downtime, cutting down operating costs and improving the reliability and safety at the required level.

\section{Acknowledgements}

This article is written within industrial research study "Development of advanced vibration monitoring technology for rolling bearing of transport and energy machines" included in the project „Establishment of TRANSPORT MECHANICAL ENGINEERING COMPETENCE CENTER”, contract No. L-KC-11-0002 with Investment and Development Agency of Latvia.

\section{References}

1. Barkov, A.V., Barkova, N.A., Nizovtsev, A.Y. (2000) Vibration-based monitoring and diagnostics of rotary machines: St. Petersburg: St. Petersburg MTU. 159 p.

2. Barkova, N.A. (2010) Optimization of HF vibration-based roller bearings diagnosing methods, from www.uran.donetsk.ua/ masters/2010/etf/prokopets/library/article5.htm

3. Measurements and tests. (1981) In: V.N. Chelomey et al. (Eds.), Vibration in Engineering: Reference book, in 6 volumes. M.: Machine-building industry, 1981. $496 \mathrm{p}$. 